\title{
Anesthesia for Surgical Correction of Coronary Artery Fistula without Extracorporeal Circulation: Case Report
}

\author{
Raquel Reis Soares, TSA 1, Leonardo Ferber Drumond 2, Leonardo Alves Araújo ${ }^{3}$, Matheus Ferber Drumond 4 , \\ Michelle Nacur Lorentz, TSA 5
}

Summary: Soares RR, Drumond LF, Araujo LA, Drumond MF, Lorentz MN - Anesthesia for Surgical Correction of Coronary Artery Fistula without Extracorporeal Circulation: Case Report.

Background and objectives: Described by Krause in $1865^{1}$, coronary artery fistula communicating with cardiac cavities, pulmonary artery, or coronary sinus is very rare. It represents $0.2 \%$ to $0.4 \%$ of congenital cardiopathies and $0.1 \%$ and $0.2 \%$ of the adult population undergoing coronary angiography ${ }^{2}$. The objective of this report is to present the anesthetic management for surgical closure of a coronary fistula in an elective procedure, considering its particularities.

Case report: This is a 59-year old male patient, whose coronary fistula was diagnosed during clinical investigation of progressive thoracic pain and long-standing dyspnea. The patient underwent surgical correction of coronary artery fistula under general anesthesia without extracorporeal circulation (ECC). The patient evolved without intercurrences, being discharged from the hospital with clinical improvement 7 days after surgery.

Conclusions: Coronary artery fistula is rare, but the anesthesiologist may be faced with this type of patient in distinct situations. Understanding its pathophysiology is important for better perioperative management of the patient, therefore improving the prognosis.

Keywords: Coronary Circulation; Coronary Artery Disease; Anesthesia, General; Fistula.

\section{INTRODUCTION}

Described in 1865 by Krause ${ }^{1}$, coronary fistula is a congenital or acquired anomaly characterized by abnormal communication between a coronary artery and a cardiac chamber, pulmonary artery, or coronary sinus ${ }^{2}$. It represents $0.2 \%$ to $0.4 \%$ of congenital cardiopathies, with a male to female ratio of 1.9:1 $1^{4}$.

Fistulas with larger shunts are related to symptoms and, in general, should be treated surgically or with hemodynamic embolization with disposable balloon. Sternotomy and open ligature with or without extracorporeal circulation (ECC) represent the most common option in more complex fistulas ${ }^{4,5}$. Its diagnosis is based, mainly, on a continuous murmur found on routine physical examination ${ }^{6}$, congestive heart failure

Received from Biocor Hospital, Nova Lima, MG, Brazil.

1. Pain Specialist, Brazilian Society for Pain Study (SBED); Acupuncture Specialist, Brazilian Medical Society for Acupuncture (SMBA); Anesthesiologist at Biocor Hospital

2. Cardiovascular Surgeon, Brazilian Society for Cardiovascular Surgery (SBCCV) and Biocor Hospital

3. R3, CET Fundação Hospitalar do Estado de Minas Gerais (FHEMIG)

4. Cardiovascular Surgeon at Biocor Hospital

5. Master's Degree, Universidade Federal de Minas Gerais (UFMG); Anesthesiologist at Biocor Hospital

Submitted on October 7, 2010

Approved on March 14, 2011.

Correspondence to:

Dra. Raquel Reis Soares

Rua da Paisagem 280/ sala 601

Vila da Serra

34000000 - Nova Lima, MG, Brazil

E-mail: raquelrsoares@globo.com physical exam, or precordial pain. With the advent of echocardiography and Doppler, non-invasive diagnosis can be performed ${ }^{7}$ using invasive procedures only to confirm and treat the disease.

\section{CASE REPORT}

- 59-year old male patient, hypertensive, former smoker, former alcoholic, admitted with history of progressive dyspnea and thoracic pain for eight years, with significant worsening in the last 12 months;

- He was taking losartan, monocordil, and simvastatin for blood pressure control;

- Myocardial scintigraphy revealed hypoperfusion in the inferoseptal region of the left ventricle;

- Echocardiography showed left atrium measuring $38 \mathrm{~mm}$ and left ventricular ejection fraction of $69 \%$, with preserved global systolic and biventricular function, and abnormal resting diastolic relaxation. It also showed subtle degenerative changes in the aortic, mitral, and tricuspid valves with mild dilation of the aortic root;

- Catheterism showed a small area of apical dyskinesia in the left ventricle compatible with apical lesion, and mild hypokinesia of the remaining myocardium, mild mitral regurgitation, and coronary arteries without significant lesions. It also showed a microfistula from the septal branch of the anterior descending coronary 
artery to the left chamber and a high output fistula from the right coronary artery to the right ventricle.

- Remaining laboratorial tests were within normal limits.

It is known that apical lesion is one of the typical manifestations of chronic Chagas' cardiomyopathy. This finding is present in up to $86.9 \%$ of cases, and it is characterized by varying degrees of thinning of myocardial fibers and occasional protrusion of the tip of the left ventricle. This lesion can also be associated with ischemic cardiopathy from steal of coronary blood flow, for example ${ }^{8,9}$.

We decided to take the patient to the operating room for open surgery for ligature of the high output fistula and correction of the left apical aneurism. It was an elective surgery under general balanced anesthesia without extracorporeal circulation.

The patient was calm and hemodynamically stable when he was admitted in the operating room after receiving $3 \mathrm{mg}$ of oral bromazepam. Monitoring was composed of cardioscope, pulse oximeter, and non-invasive blood pressure. Venipuncture was performed with an $18 \mathrm{G}$ catheter, proceeding with sedation with $3 \mathrm{mg}$ of midazolam. The left radial artery was punctured with a $20 \mathrm{G}$ catheter. He had mean blood pressure of $70 \mathrm{mmHg}$, heart rate of $80 \mathrm{bpm}$, and $\mathrm{SpO}_{2} 96 \%$. Anesthetic induction was performed with fractionated doses of fentanyl (up to $15 \mu \mathrm{g} \cdot \mathrm{kg}^{-1}$ ), midazolam (up to $7 \mathrm{mg}$ ), and atracurium $\left(0.5 \mathrm{mg} . \mathrm{kg}^{-1}\right)$ for neuromuscular blockade and tracheal intubation. Hemodynamic repercussions during anesthetic induction were not observed. Monitoring was completed with gas analyzer, esophageal temperature, and serial laboratorial tests. The left subclavian vein was punctured with a $16 \mathrm{G}$ long-term catheter without intercurrences, and central venous pressure, which initially was $13 \mathrm{mmHg}$, was monitored. Anesthesia was maintained with inhalational isoflurane, maintaining MAC from 0.5 to 1 , according to patient's needs.

After sternotomy and exposure of the surgical field, a dilated right coronary artery in all its extension (diameter > $4 \mathrm{~mm}$ ) was observed. The fistula was clamped for evaluation of right atrial and pulmonary artery troncus blood gases, comparing the results before and after clamping the fistula. Blood gases performed before clamping showed saturation in the pulmonary artery troncus $5 \%$ above the right atrial saturation (pulmonary artery saturation $80.4 \%$ and right atrial saturation $75.4 \%$ ), revealing shunt of arterial blood into the right ventricle. After clamping the fistula, saturation on the pulmonary artery troncus blood was $5 \%$ below right atrial saturation (pulmonary artery saturation, $71.0 \%$, and right atrial saturation, $75.8 \%$ ), which is expected due to drainage of the coronary sinus into the right atrium, close to the tricuspid ring.

Follow-up of blood gases in both cardiac chambers revealed the oximetry data jump, indicating correction of the problem, as previously demonstrated. After the oximetry data jump was observed and the blood vessel was identified, double ligature of the fistula was performed and the left ventricular apical aneurysm was corrected.

Due to the small caliber, the fistula into the left ventricle was not clamped. The patient remained hemodynamically stable, without electrocardiographic changes.
The procedure evolved without intercurrences, and the patient was discharged from the intensive care unit (ICU) on the second postoperative day, being discharged from the hospital 7 days after the surgery on the same preoperative medication.

\section{DISCUSSION}

Coronary arterial fistulas, congenital or acquired, in which there is a mixture of venous and arterial blood inside one of the cardiac chambers, large vessel, or other structure bypassing myocardial capillary circulation are rare.

Approximately $10 \%$ to $30 \%$ of patients with coronary fistula have associated anomalies, such as persistence of patent ductus arteriosus, tetralogy of Fallot, interventricular communication, and acquired cardiopathies ${ }^{10-14}$.

This anomaly is a potential cause of sudden death in children and athletes, and most reports are made by pediatricians and pathologists. In reviewing the literature, the finding of a murmur on physical examination leads to the diagnosis and surgery for correction of coronary fistulas.

Coronary artery fistula is a rare disease whose diagnosis can be easily made with echocardiography ${ }^{6}$. Despite this, it is the main congenital anomaly of the coronary arteries that change cardiac hemodynamic parameters.

The etiology of coronary artery fistulas is unknown. It has been suggested that they result from persistence of intratrabecular spaces though which the myocardium is supplied during intrauterine life and that are reduced after birth ${ }^{15}$. Approximately $75 \%$ of coronary artery fistulas are small and clinically silent. They are twice as frequent in the right coronary artery (55\%-60\%) and most times (90\%) they drain to the small circulation (right ventricle $45 \%$; right atrium $25 \%$; pulmonary artery $15 \%-20 \%$; and coronary sinus $7 \%{ }^{16}$.

Small caliber fistulas draining into the pulmonary artery are more frequent in left coronary artery and in $4 \%$ to $5 \%$ of the cases involvement of the two coronary arteries is seen. Acquired coronary artery fistulas are secondary to complications of some invasive procedure or trauma ${ }^{17}$.

Currently, diagnosis of coronary artery fistulas can be done with non-invasive techniques such as coronary multislice angiotomography and in some cases, 3D MRI, which, besides showing the anatomy of the fistula, demonstrates its relationship with important cardiac structures ${ }^{14}$. In our field, coronary angiography still is the gold standard in the diagnosis and hemodynamic evaluation of this anomaly ${ }^{18,19}$.

Usually, the onset of symptoms is late, and approximately $75 \%$ of patients show the first symptoms after 40 years of age ${ }^{16}$. Patients can develop congestive heart failure with dyspnea, as well as signs of myocardial ischemia ${ }^{6}$.

Pathophysiology and clinical picture depend of the magnitude of the blood flow through the fistula and its location. Most cases described in literature concerns the right chambers, with varying degrees of pulmonary hyperflow. Clinical manifestations include heart failure or exertional dyspnea. Ischemia secondary to fistulas can be secondary to steal of 
coronary blood flow, embolus, or thrombosis with precordial pain or signs of ischemia on conventional electrocardiogram or exercise ECG. Steal syndrome in patients without coronary artery disease is due to greater reduction of coronary arterial resistance compared to pulmonary resistance during exercise. The patient reported here had right and left coronary artery fistulas and precordial pain and dyspnea in agreement with the clinical picture described.

Most patients are asymptomatic, although dyspnea, angina, acute myocardial infarction, pulmonary hypertension, arrhythmias, bacterial endocarditis, and sudden death may occur. Management is controversial; however, symptomatic patients or with significant shunts may be treated with surgical or percutaneous closure of the fistula ${ }^{6}$.

Surgical indication depends on the presence of clinical symptoms, such as heart failure and myocardial ischemia, or presence of significant shunt ${ }^{4}$. Available options include surgical or percutaneous closure, and the latter is a safe and effective alternative with trained teams ${ }^{20,21}$.

The presence of associated cardiac anomalies indicates surgical correction to treat the remaining pathologies. Closure of the fistula tract can be performed through intracavitary approach by ligature of the endocardial fistula, or extracavitary approach, by ligature of epicardial fistula, or continuous suture, with or without extracorporeal circulation.

In a review of 27 years of experience, Kamiya et al. ${ }^{22}$ demonstrated that only $20 \%$ of the cases were operated without ECC. Surgical mortality of closure of isolated coronary fistula is only $1 \%$, but recurrence or presence of residual fistula that only appears when wider caliber fistula is closed can occur ${ }^{23}$. Asymptomatic patients should be under clinical follow-up. And regardless of the therapy long-term follow-up of these patients is mandatory ${ }^{23}$.

The case presented here is about a patient with involvement of two coronary arteries; however, the fistula in the right coronary artery had high output, which was responsible for the symptoms and motivated the surgery, while the fistula in the left coronary artery was small and without clinical repercussion. The apical aneurism was also corrected. The patient was operated without extracorporeal circulation to reduce systemic inflammatory response and surgical trauma, especially since he was asthmatic.

The present case report is important because it addresses the type of patient that can seek the anesthesiologist for surgical correction of coronary artery fistula, percutaneous closure of a coronary artery fistula in a hemodynamics laboratory, and for other non-cardiac surgeries, elective or urgent, in case of submission to clinical control.

These are high risk patients for cardiovascular decompensation, coronary ischemia, in addition to pulmonary hyperflow, favoring the development of pulmonary hypertension. Hemodynamic stability, normovolemia, and control of anesthetic plane are extremely important to avoid sympathetic hyperactivity. Intraoperative ST- segment changes, myocardial infarction, fistula dissection, coronary spasm, changes in cardiac rhythm, and embolization during open or percutaneous surgeries have been described, and the anesthesiologist should be prepared to treat these complications. 